\title{
Radiological Mimics of Popliteal Cysts: An Algorithmic Approach Using US and MRI to Identify the Potentially Malignant Lesions: Case Series
}

\author{
Andrae Blanchard', Asad A. Naqvi², Zain Badar ${ }^{3}$, Hema Choudur ${ }^{*}$ \\ ${ }^{1}$ University of Western Ontario, London, Canada \\ 2Department of Diagnostic Imaging, Queen's University, Kingston, Canada \\ ${ }^{3}$ Department of Radiology, State University of New York Upstate Medical University, Syracuse, USA \\ ${ }^{4}$ McMaster University, Hamilton, Canada \\ Email:ablanch5@gmail.com, anaqvi@qmed.ca,drzainbadar@gmail.com, ‘choudur@hhsc.ca
}

Received 27 February 2016; accepted 30 May 2016; published 2 June 2016

Copyright (C) 2016 by authors and Scientific Research Publishing Inc.

This work is licensed under the Creative Commons Attribution International License (CC BY). http://creativecommons.org/licenses/by/4.0/

(c) (i) Open Access

\begin{abstract}
Background: Popliteal cysts are common and present as asymptomatic lumps in the medial popliteal fossa. Some have complex internal characteristics such as septa and loose-bodies. However, not all are popliteal cysts and can be aggressive. These lesions need to be differentiated by the absence of the communicating neck with the joint on ultrasound. Presence of Doppler flow of non-communicating cysts requires further evaluation on MRI, prior to performing a biopsy. Using a case series, we propose an algorithmic approach that is simple and will help identify the malignant lesions and institute appropriate management. Case-Presentation: Popliteal Cyst: On ultrasound: characteristic neck communicating with knee joint. Synovial Sarcoma: Gadolinium enhancement, with areas of low-, iso- and hyper-intense signal to fat on T2. Synovial-Osteochondromatosis: Non-mineralized: T1-low/intermediate intensity; T2-high intensity. Mineralized type: low intensity on T1 \& T2. Thrombosed Popliteal Aneurysm: Lamellated appearance-high/low signal intensity on T2. MyxoidLiposarcomas: Inhomogeneous appearance; homogenous with gadolinium. Usually require a biopsy for diagnosis. Conclusion: The cystic lesions in the medial aspect of the popliteal fossa can be misdiagnosed. Our article reiterates the importance of the communicating neck that separates popliteal cysts from other mimics. We have proposed an algorithm to identify these mimics.
\end{abstract}

\section{Keywords}

Popliteal Cyst, Malignant Lesions, Mimics, Algorithmic Approach

\footnotetext{
${ }^{*}$ Corresponding author.
}

How to cite this paper: Blanchard, A., Naqvi, A.A., Badar, Z. and Choudur, H. (2016) Radiological Mimics of Popliteal Cysts: An Algorithmic Approach Using US and MRI to Identify the Potentially Malignant Lesions: Case Series. Open Journal of Clinical Diagnostics, 6, 7-12. http://dx.doi.org/10.4236/ojcd.2016.62002 


\section{Background}

A popliteal cyst is an abnormal fluid filling of the semimembranosus-gastrocnemius bursa [1]. In adults, popliteal cysts are often associated with knee joint pathology and have a communicating neck between the bursa and the synovial fluid of the knee [2] [3]. A 5\% - 18\% general prevalence rate on MRI and a 40\% - 42\% prevalence rate by ultrasound is reported in literature on ultrasound for deep venous thrombosis of the lower extremity [2].

The communicating neck of the popliteal cyst is along the line of least resistance-between heads of medial gastrocnemius and semimembranosus and is the chief identifying feature of a popliteal cyst. This is usually well delineated by Ultrasound. Once identified, no further imaging would be necessary. The popliteal cysts lack Doppler flow unless they are infected or are inflammatory. However, the presence of Doppler flow also raises the possibility of a potentially aggressive lesion and an MRI would be deemed necessary. If there is gadolinium enhancement, the patient would undergo a biopsy to exclude an aggressive lesion.

The differential diagnosis of posterior knee lesions is broad and includes cystic lesions (other bursae, meniscal cysts, ganglion cysts and popliteal cysts). Unlike other cysts, the popliteal cysts have a communicating neck with the joint, which is a chief differentiating feature (on ultrasound and MRI) not seen with other lesions mimicking popliteal cysts, including: solid tumors (liposarcoma, synovial sarcoma, synovial osteochondromatosis), or vascular abnormalities (popliteal artery and vein thrombosis) [4] [5].

In this article, we review the chief US and MR characteristics of popliteal cysts and some biopsy proven cases of mimics of popliteal cysts in each of the broad categories of cystic tumors, vascular lesions and synovial based cystic lesions. We propose an algorithm to separate the potentially aggressive lesions utilizing MRI. A timely biopsy of such lesions will facilitate early management, especially lesions such as synovial sarcomas that are asymptomatic early in the course of the disease. Such an algorithmic approach will prevent misdiagnosis and delay in appropriate treatment of the aggressive lesions. Such an algorithm in a flow chart format, Figure 1, has not been described, to the best of our knowledge in the literature.

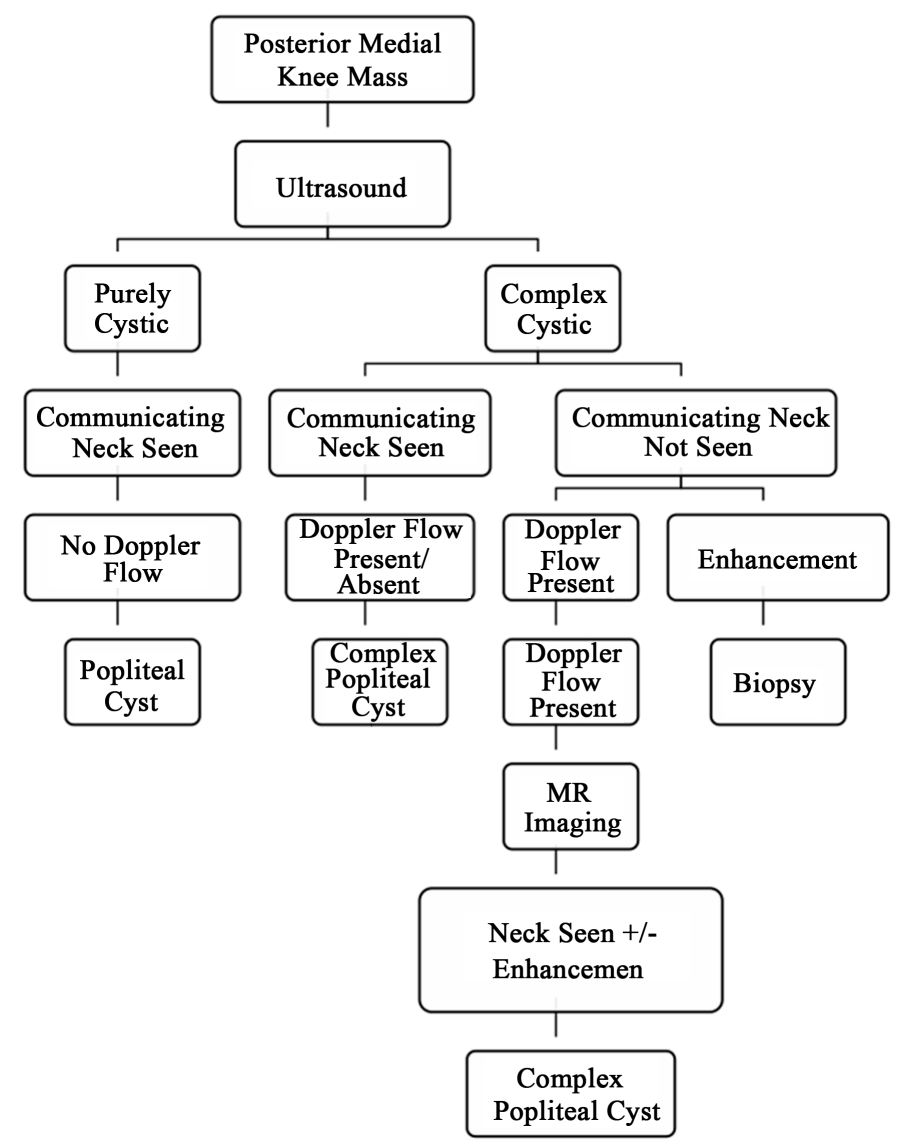

Figure 1. Diagnostic imaging approach to posteromedial knee (medial popliteal fossa) masses. 


\section{Popliteal Cyst}

Most popliteal cysts are asymptomatic and are discovered incidentally, usually in adults between 35 - 70 and their incidence on MRI ranges from 47\% - 19\% [6]. Common signs and symptoms are posterior knee pain, knee stiffness, and swelling behind the knee. Complications, although rare, are due to enlargement or rupture. Enlarged cysts may present with erythema, or cause compressive symptoms such as distal edema, nerve entrapment, or a compartment like syndrome. Ruptured cysts can resemble deep vein thrombosis with tenderness and erythema of the calf. Treatment of symptomatic cysts can be accomplished with image guided aspiration and intraarticular injection of glucocorticoids, which benefits two-thirds of patients [3].

Due its superficial location, popliteal cysts are readily demonstrated on ultrasound. They are visualized sonographically as typically fluid filled, anechoic structures with a communicating neck between the medial gastrocnemius and semimembranosus tendons. Popliteal cysts may show complex features such as cartilagenous or osseous intra-articular loose bodies, Figure 2, and they may be multi-lobulated, demonstrate synovial proliferation, or contain hemorrhage [6] [7]. Doppler flow may indicate inflammation or infection or is suggestive of another diagnosis. On MRI, popliteal cysts show fluid signal intensity on all sequences unless infection or hemorrhage is present, in which case increased signal intensity will be seen on T1 images. Rupture or leakage will show as high signal edema on fat suppressed T2 images [4]. Osseous or cartilagenous loose bodies will also be visualized on MRI.

\section{Synovial Sarcoma}

Synovial sarcoma accounts for approximately $10 \%$ of soft tissue sarcomas. Men and women are affected equally and the mean age of presentation is 32. They occur most often in the extremities, with $70 \%$ of all cases occurring in the lower limbs. These masses are generally slow growing and patients seek medical attention, a mean of 2.5 years after initiation of symptoms. Synovial sarcoma is thought to originate from primitive mesenchymal cells that after differentiation resemble synovial cells. Synovial sarcomas do not commonly arise in intra-articular locations but rather near them. They have a 50\% recurrence rate within two years and they metastasize mainly to the lung [8]. On MRI, lesions are typically found to be greater than $5 \mathrm{~cm}$, near a joint, and adjacent to bone. T2 weighted images, Figure 3, often display areas that are low, iso-, and hyper-intense to fat ("triple sign") [9]. Smaller lesions may be found more peripherally, have smoother contours, have uniform signal characteristics, and lack local invasion.

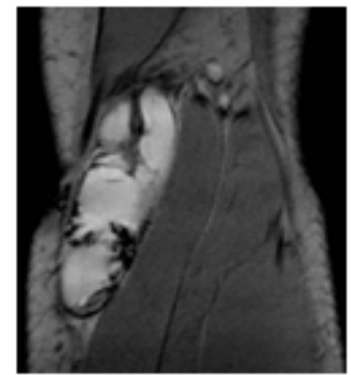

(a)

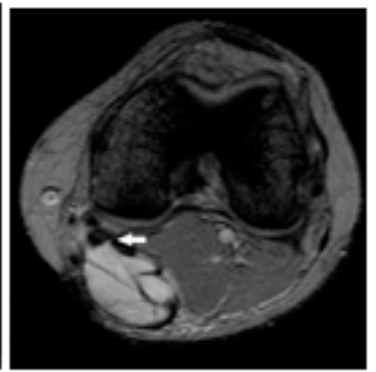

(b)

Figure 2. MR Imaging of a complex popliteal cyst. (a) Sagittal (b) Axial T2 gradient sequences: note the septations and nodular synovial thickening. The communicating neck with the joint is clearly depicted on the axial sequence.

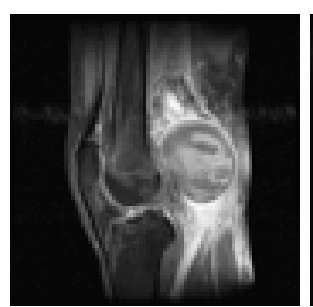

(a)

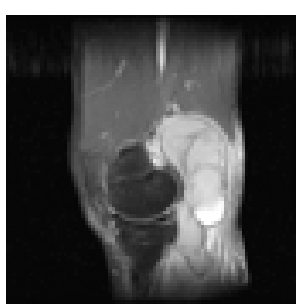

(b)

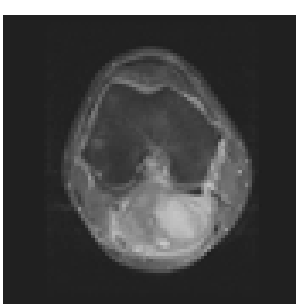

(c)

Figure 3. Synovial sarcoma. (a) T2 fat sat sagittal; (b) T2 fat sat sagittal; (c) T2 fat sat Axial sequences. 


\section{Synovial Osteo Chondromatosis}

Synovial osteochondromatosis is a disorder of the synovial lining characterized by cartilage hyperplasia and synovial neoplasia resulting in the formation of cartilaginous nodules or chondromas. Most of these eventually calcify to become osteochondromas and a small percentage will undergo ossification. Nodules are released into the joint space forming loose bodies, which can cause mechanical joint pain, joint locking, and joint effusions. Synovial osteochondromatosis has an incidence of 1 in 10,000, is mono-articular in $95 \%$ of cases and $50 \%$ of cases involve the knee. On MRI, three appearances have been described: type A, which contain non-mineralized lesions; type B, which contain mineralized lesions; and type C, which contain adipose tissue [10]. The non-mineralized (chondroma) type is found in 16\% of cases and displays low/intermediate intensity on T1 and high signal intensity on T2 and may be difficult to distinguish from synovial fluid or mass. In this case, contrast can be helpful as these lesions typically show rim enhancement as they have a vascular supply from the synovium [11]. The mineralized type accounts for $75 \%$ of cases and is of low signal intensity on T1 and $\mathrm{T} 2$ sequences, Figure 4. Nodules in type C are isointense to fat and show high signal on T1 and intermediate signal on T2.

\section{Thrombosed Popliteal Artery Aneurysm}

Popliteal artery is associated with large vessel aneurysms. Fifty percent are bilateral and thirty-five percent of patients are asymptomatic at diagnosis. Symptoms, when they occur, are due to thrombosis, rupture, or local compressive symptoms. Thrombosis can cause claudication or acute limb ischemia. Compressive symptoms include localized pain or a sense of fullness behind the knee [12]. Rupture that causes severe pain occurs in less than $5 \%$ of cases [13]. Excision is indicated when patients present with acute limb ischemia or with patent popliteal aneurysms greater than $2 \mathrm{~cm}$. On MRI, the classic feature is a lamellated appearance consisting of high and low signal intensity due to multilayered mural thrombus. The presence of pulsation artifact can also be useful [5] [14].

Figure 5 and Figure 6 show axial T2 and T2 fat saturated axial images demonstrate the lack of communicating neck with the joint as seen with a Baker’s cyst. The lesion lies in the region of the popliteal artery.

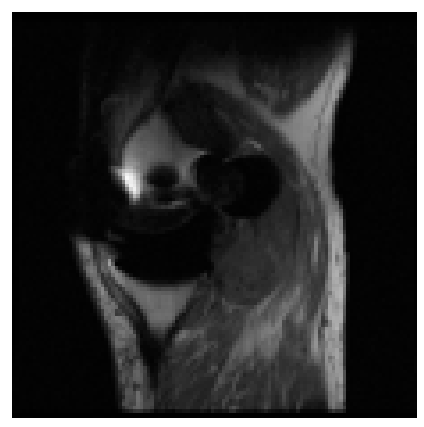

(a)

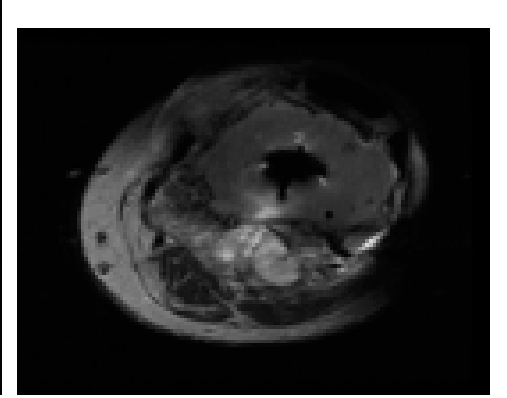

(b)

Figure 4. Synovial Osteochondromatosis in a patient following knee arthroplasty. Note the low T1 and T2 mineralized foci within the lesion (a) T1 sagittal; (b) T2 Axial sequences.

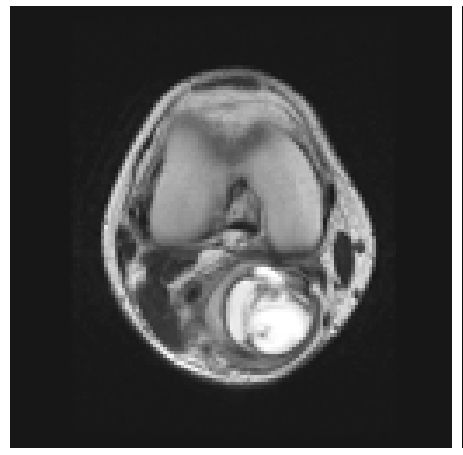

(a)

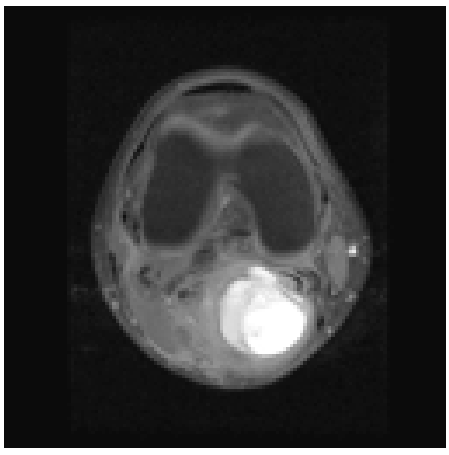

(b)

Figure 5. Thrombosed popliteal artery aneurysm. (a) T2 axial; (b) T2 fat sat axial sequences. 


\section{Myxoid Liposarcoma}

Liposarcoma is the second most common soft tissue sarcoma in adults, presenting typically between the age of 40 and 60, and commonly arising in deep muscle masses. Around $40 \%$ of these tumours occur in the lower extremity [15]. Morphologically liposarcomas can be classified as well differentiated, myxoid/round, and pleomorphic. Myxoid tumours comprise 50\% of liposarcomas and have intermediate malignant potential and local recurrence is common without full excision. The most common presentation is a painless enlarging mass. On MR imaging, Figure 7, they typically have an inhomogeneous appearance, may appear cystic, and they typically have a fat content of less than $25 \%$, making them potentially difficult to distinguish from popliteal cysts.

\section{Conclusions}

Ultrasound is capable of defining solid and cystic lesions. Often, solid lesions such as myxoid liposarcoma and synovial sarcoma appear as complex cysts on ultrasound and may be mistaken for a popliteal cyst when they occur in the medial aspect of the popliteal fossa. The presence of Doppler flow ultrasound necessitates further investigation with MRI to identify potentially aggressive lesions. The presence of a communicating neck is reassuring for a popliteal cyst. However, if this neck is not identified, it would be prudent to further assess with a post-gadolinium MRI and identify the potential aggressive lesion. A previous study evaluating the accuracy of ultrasound in popliteal cysts found that the two lesions identified on MRI that were not popliteal cysts, were initially misdiagnosed as popliteal cysts [6]. The authors found that neither lesion would have been misdiagnosed if a communicating neck was demonstrated on ultrasound. The presence of Doppler flow necessitates further investigation with MRI to identify potentially aggressive lesions, though inflammatory or infectious changes of popliteal cysts can increase the Doppler flow. Popliteal cysts can show complex features when they contain intra-articular loose bodies, however this may also be seen with synovial osteochondromatosis. Thus while ultrasound may be sensitive and specific in the diagnosis of popliteal cysts, it may not be so for the more unusual lesions presented here.

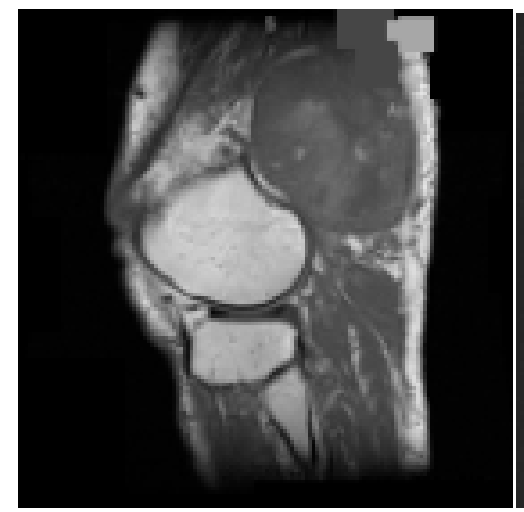

(a)

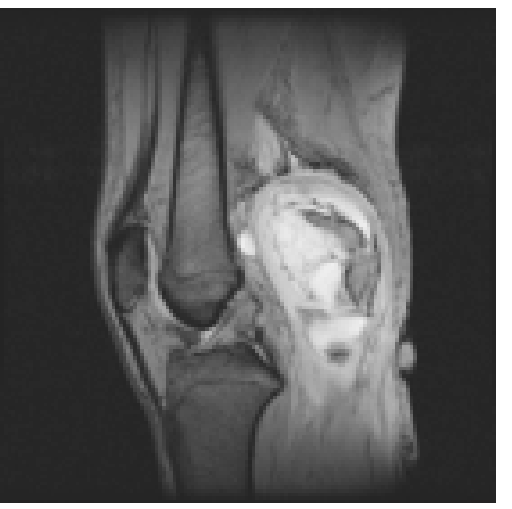

(b)

Figure 6. Thrombosed popliteal artery aneurysm. (a) T1 sagittal; (b) Gradient T2 sagittal sequences.

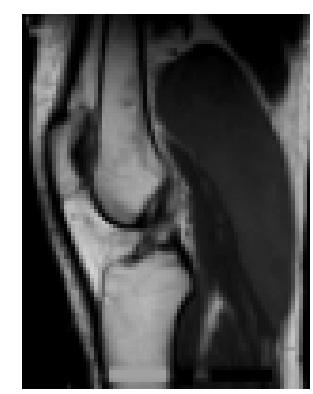

(a)

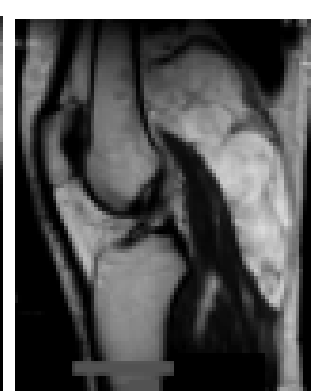

(b)

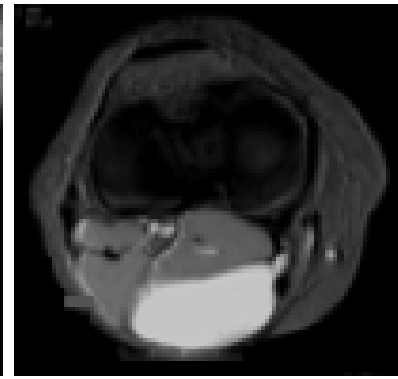

(c)

Figure 7. Myxoid Liposaroma. (a) T1 sagittal; (b) T2 sagittal; (c) T2 axial sequences. A pseudoneck is seen at its anteromedial aspect mimicking a popliteal cyst. 
We propose that those cysts on ultrasound that do not demonstrate a communicating neck, have Doppler flow, or have a complex cystic structure without the identifiable communication neck be further analyzed with post gadolinium MRI. Either of the three mentioned features or a combination of the three features would be an indication for MRI. If the non-contrast axial MRI depicts the communicating neck irrespective whether the cyst is simple or complex, a diagnosis of a popliteal cyst is made. Absence of the neck with complex features on the unenhanced MRI warrants gadolinium administration whether or not Doppler flow is identified on ultrasound. Furthermore we recommend that those lesions that do not demonstrate a communicating neck but enhance on gadolinium be biopsied.

\section{References}

[1] Bickel, W.H., Burleson, R.J. and Dahlin, D.C. (1956) Popliteal Cyst: A Clinicopathological Survey. The Journal of Bone \& Joint Surgery, 38-A, 1265-1274.

[2] Fritschy, D., Fasel, J., Imbert, J.C., Bianchi, S., Verdonk, R. and Wirth, C.J. (2006) The Popliteal Cyst. Knee Surgery, Sports Traumatology, Arthroscopy, 14, 623-628. http://dx.doi.org/10.1007/s00167-005-0028-z

[3] Handy, J.R. (2001) Popliteal Cysts in Adults: A Review. Seminars in Arthritis and Rheumatism, 31, 108-118. http://dx.doi.org/10.1053/sarh.2001.27659

[4] Marra, M.D., Crema, M.D., Chung, M., Roemer, F.W., Hunter, D.J., Zaim, S., Diaz, L. and Guermazi, A. (2008) MRI Features of Cystic Lesions around the Knee. Knee, 15, 423-438. http://dx.doi.org/10.1016/j.knee.2008.04.009

[5] McCarthy, C.L. and McNally, E.G. (2004) The MRI Appearance of Cystic Lesions around the Knee. Skeletal Radiology, 33, 187-209. http://dx.doi.org/10.1007/s00256-003-0741-y

[6] Ward, E.E., Jacobson, J.A., Fessell, D.P., Hayes, C.W. and van Holsbeeck, M. (2001) Sonographic Detection of Baker’s Cysts: Comparison with MR Imaging. AJR American Journal of Roentgenology, 176, 373-380. http://dx.doi.org/10.2214/ajr.176.2.1760373

[7] Helbich, T.H., Breitenseher, M., Trattnig, S., Nehrer, S., Erlacher, L. and Kainberger, F. (1998) Sonomorphologic Variants of Popliteal Cysts. Journal of Clinical Ultrasound, 26, 171-176. http://dx.doi.org/10.1002/(SICI)1097-0096(199803/04)26:3<171::AID-JCU12>3.0.CO;2-E

[8] Bakri, A., Shinagare, A.B., Krajewski, K.M., Howard, S.A., Jagannathan, J.P., Hornick, J.L. and Ramaiya, N.H. (2012) Synovial Sarcoma: Imaging Features of Common and Uncommon Primary Sites, Metastatic Patterns, and Treatment Response. AJR American Journal of Roentgenology, 199, W208-W215. http://dx.doi.org/10.2214/AJR.11.8039

[9] Jones, B.C., Sundaram, M. and Kransdorf, M.J. (1993) Synovial Sarcoma: MR Imaging Findings in 34 Patients. AJR, 161, 827-830. http://dx.doi.org/10.2214/ajr.161.4.8396848

[10] Kramer, J., Recht, M., Deely, D.M., Schweitzer, M., Pathria, M.N., Gentili, A., Greenway, G. and Resnick, D. (1993) MR Appearance of Idiopathic Synovial Osteochondromatosis. Journal of Computer Assisted Tomography, 17, 772-776. http://dx.doi.org/10.1097/00004728-199309000-00020

[11] McKenzie, G., Raby, N. and Ritchie, D. (2008) A Pictorial Review of Primary Synovial Osteochondromatosis. European Radiology, 18, 2662-2669. http://dx.doi.org/10.1007/s00330-008-1024-8

[12] Dawson, I., Sie, R.B. and van Bockel, J.H. (1997) Atherosclerotic. Popliteal Aneurysm. British Journal of Surgery, 84, 293-299. http://dx.doi.org/10.1002/bjs.1800840304

[13] Vermilion, B.D., Kimmins, S.A., Pace, W.G. and Evans, W.E. (1981) A Review of One Hundred Forty-Seven Popliteal Aneurysms with Long-Term Follow-Up. Surgery, 90, 1009-1014.

[14] Butler, M.G., Fuchigami, K.D. and Chako, A. (1996) MRI of Posterior Knee Masses. Skeletal Radiology, 25, 309-317. http://dx.doi.org/10.1007/s002560050087

[15] Munk, P.L., Lee, M.J., Janzen, D.L., Connell, D.G., Logan, P.M., Poon, P.Y. and Bainbridge, T.C. (1997) Lipoma and Liposarcoma: Evaluation Using CT and MR Imaging. AJR American Journal of Roentgenology, 169, 589-594. http://dx.doi.org/10.2214/ajr.169.2.9242783 